\title{
Components of Essential Oil from Woods of Prunus mume Sieb. et Zucc.
}

\author{
Hirotoshi Utsunomiya ${ }^{1}$, Jyunichi Kawata ${ }^{2}$, Wato ChanokI $^{2}$, Nozomu Shirakawa ${ }^{2}$ \\ and Mitsuo Mirazawa ${ }^{2 *}$ \\ ${ }^{I}$ Department of Pathology, Wakayama Medical University \\ (811-1 Kimiidera, Wakayama 641-0012, JAPAN) \\ ${ }^{2}$ Department of Applied Chemistry, Faculty of Science and Engineering, Kinki University \\ (3-4-1, Kowakae, Higashiosaka-shi, Osaka 577-8502, JAPAN)
}

Edited by K. Kubo, Health Sci. Univ. Hokkaido, and accepted July 6, 2005 (received for review June 20, 2005)

\begin{abstract}
The compositions of the essential oil from woods of Prunus mume Sieb. et Zucc. (ume), have been investigated by capillary GC and GC/MS. The oil was found to contain 97 components, representing $92.41 \%$ of the total oil. The main constituents were $6,10,14-$ trimethyl-2-pentadecanone $(15.83 \%), \alpha$-acorenol $(9.36 \%),(\mathrm{Z})$ - $\alpha$-bisabolene $(7.49 \%)$, benzaldehyde (3.87\%), isopropyltiglate (3.84\%), terpinen-4-ol $(3.41 \%)$.

Key words: Prunus mume Sieb. et Zucc., essential oil, 6,10,14-trimethyl-2-pentadecanone, $\alpha$-acorenol, (Z)- $\alpha$-bisabolene, benzaldehyde
\end{abstract}

\section{Introduction}

Japanese apricot (Prunus mume Sieb. et Zucc., ume) is a deciduous tree of Rosaceae family. The tree have been carried to Japan from China in the end of 7th century. The fruits have a history of the used of tradition foods, umeboshi or pickled Japanese apricot, umeliqueur, ume-juice, ume-jam, and as well as folk medicine for alleviating fever, cough and intestinal disorders in Japan. Therefore, there are a lot of reports of constitution from the fruits. From the fruits, some organic acids as acidity compounds have been isolated, such as malic acid, citric acid, oxalic acid, succinic acid (1-3). In addition, many volatile compounds have been identified (4-8). Chu-Chin Chen has reported 92 compounds identified from fruits and the main components were benzaldehyde, benzyl alcohol. Arikawa has reported 52 compounds identified from ume-liqueur by steam distillation and headspace. Ishida also reported 48 compounds were identified from umeboshi. On the other hand, the woods of $P$. mume are liked as a plant for appreciation by the Japanese and have some flavonoids (9-11). However, there is no report of the essential oil from woods of $P$. mume. In this paper, essential oil from woods of $P$. mume was investigated using GC and GC/MS.

\section{Experimental}

\subsection{Plant}

The samples, woods of $P$. mume, were collected from Minabe-town (Wakayama, Japan) in July 2004.

\section{$\mathbf{2} \cdot 2$ Isolation of the Volatile Oil}

Woods $(15 \mathrm{~kg})$ of $P$. mume were hydrodistilled with a Likens-Nickerson-type apparatus using diethyl ether to yield $0.015 \%$ of the yellowish green oil, which was dried over anhydrous sodium sulfate prior to analyses.

\subsection{Gas Chromatography (GC)}

GC was carried out using a Agilent Technologies $6890 \mathrm{~N}$, fitted with a flame ionization detector (FID) on

\footnotetext{
*Correspondence to: Mitsuo MiYaZawa, Department of Applied Chemistry, Faculty of Science and Engineering, Kinki University, 3-4-1, Kowakae, Higashiosaka-shi, Osaka 577-8502, JAPAN

E-mail: miyazawa@apch.kindai.ac.jp
} 
a non-polar capillary column HP-5 (J\&W Scientific; 30 $\mathrm{m} \times 0.32 \mathrm{~mm}$ i.d., film thickness $0.25 \mu \mathrm{m})$. The oven temperature was programmed from $40^{\circ} \mathrm{C}$ to $240^{\circ} \mathrm{C}$ at a rate of $4{ }^{\circ} \mathrm{C} / \mathrm{min}$ and held at $240^{\circ} \mathrm{C}$ for $5 \mathrm{~min}$. The injector and detector temperatures were $240^{\circ} \mathrm{C}$ and $280^{\circ} \mathrm{C}$. The flow rate of the carrier gas $(\mathrm{He})$ was $1.8 \mathrm{~mL} / \mathrm{min}$.

\subsection{Gas Chromatography-Mass Spectrome- try (GC-MS)}

The GC-MS analysis was carried out using a Hewlett Packard model 5890 GC with a Hewlett Packard 5972A MS. The capillary column was a nonpolar column DB$5 \mathrm{MS}$ (J\&W Scientific; $30 \mathrm{~m} \times 0.25 \mathrm{~mm}$ i.d., film thickness $0.25 \mu \mathrm{m}$ ). The oven temperature was programmed from $60^{\circ} \mathrm{C}$ to $240^{\circ} \mathrm{C}$ at a rate of $2{ }^{\circ} \mathrm{C} / \mathrm{min}$ and held at $240^{\circ} \mathrm{C}$ for $5 \mathrm{~min}$ and the flow rates of carrier gas (He) was $0.679 \mathrm{~mL} / \mathrm{min}$. The injector and detector temperatures were 240 and $280^{\circ} \mathrm{C}$ respectively, with the actual temperature in the MS source reaching approximately $180^{\circ} \mathrm{C}$ and the ionization voltage $70 \mathrm{eV}$.

\subsection{Identification of Constituents}

The components of essential oils were identified by direct comparison of their mass spectral pattern and retention index (RI) with those published in the literature (12).

\section{Result and Discussion}

The yield of oil obtained by steam distillation from the woods of $P$. mume was $0.015 \%(\mathrm{w} / \mathrm{w})$. The oil has a characteristic odor, which is a very woody with herbal and minty odor undertones. The oil was analyzed on GC and GC-MS, of which gas chromatogram was shown in Fig. 1. The percentage composition and retention index (RI) of the oil components were listed in Table 1. The oil was revealed the presence $97 \mathrm{com}-$ ponents, representing $92.41 \%$ of the total oil. The main components in the oil were 6,10,14-trimethyl-2pentadecanone $(15.83 \%), \alpha$-acorenol $(9.36 \%),(\mathrm{Z})-\alpha$ bisabolene $(7.49 \%)$, benzaldehyde $(3.87 \%)$, isopropyltiglate $(3.84 \%)$, terpinen-4-ol (3.41\%), $\alpha$-cis-belgamotene $(2.91 \%)$, nonanal $(2.28 \%)$ and heptadecane $(1.84 \%)$. Classification of the essential oil was listed in Table 2. The oil included 5 monoterpenoids (3.98\%), 30 sesquiterpenoids $(35.30 \%), 1$ diterpenoid $(0.19 \%)$, 55 aliphatic miscellaneous (46.35\%), 6 aroma compounds $(6.55 \%)$. Its aroma compounds were less than some previous report of fruits oils and the woods oil contained rich chain compounds and mono-, sesqui-terpenoids. These components seemed to play an important role in the characteristic aroma of woods of $P$. mume.

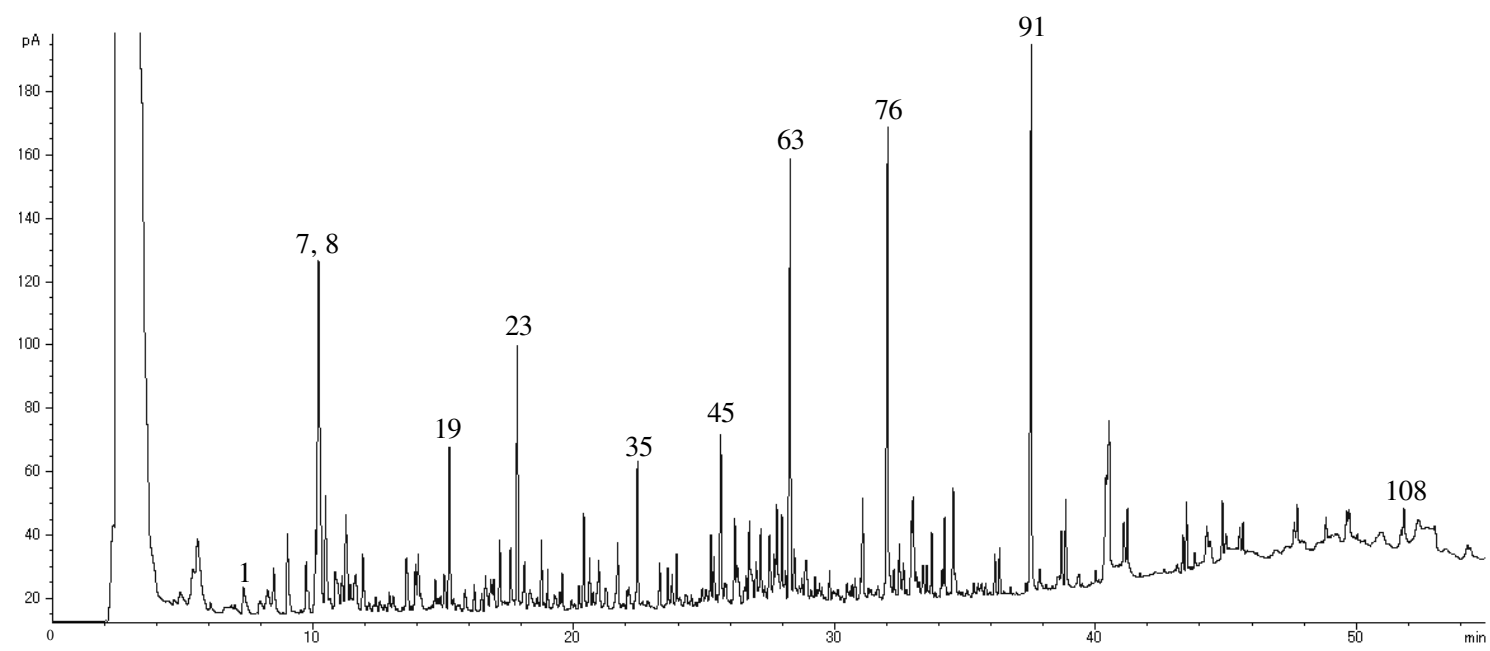

Fig. 1 Gas Chromatogram of the Essential Oil from Woods of Prunus mume. HP-5 (30 m $\times 0.32 \mathrm{~mm}$ i.d., film thickness $0.25 \mu \mathrm{m}$ ); flow rate of a carry gas $(\mathrm{He}), 1.8 \mathrm{~mL} / \mathrm{min}$; oven temperature, $40-240{ }^{\circ} \mathrm{C}$ at $4{ }^{\circ} \mathrm{C} / \mathrm{min}$ and held at $240{ }^{\circ} \mathrm{C}$ for $5 \mathrm{~min}$. 
Table 1 Components of the Essential Oil from Woods of Prunus mume.

\begin{tabular}{|c|c|c|c|c|c|c|c|}
\hline No. & RI & Compound & Peak area $(\%)$ & No. & RI & Compound & Peak area $(\%)$ \\
\hline 1 & 849 & 2-hexenone & 0.10 & 59 & 1481 & oxide calamenene & 0.30 \\
\hline 2 & 885 & $p$-xylene & $\operatorname{tr}$ & 60 & 1485 & $\alpha$-zigiberene & 1.01 \\
\hline 3 & 900 & heptanal & $\operatorname{tr}$ & 61 & 1488 & $\beta$-selinene & $\operatorname{tr}$ \\
\hline 7 & 948 & benzaldehyde & 3.87 & 62 & 1492 & $\alpha$-muurolene & 1.14 \\
\hline 8 & 952 & isopropyltiglate & 3.84 & 63 & 1498 & (Z)- $\alpha$-bisabolene & 7.49 \\
\hline 9 & 965 & 1-octen-3-ol & $\operatorname{tr}$ & 64 & 1500 & pentadecane & 0.32 \\
\hline 10 & 969 & 6-methyl-5-hepten-2-one & 0.31 & 65 & 1504 & $\beta$-bisabolene & 0.52 \\
\hline 11 & 974 & 2-pentyl furan & 1.13 & 66 & 1512 & $\delta$-cadinene & 0.81 \\
\hline 12 & 988 & 1-decene & $\operatorname{tr}$ & 67 & 1517 & $\gamma$-dehydro-ar-himachalene & 0.13 \\
\hline 13 & 996 & $(2 E, 4 E)$-heptadienal & 0.16 & 68 & 1519 & (Z)-nerolidol & $\operatorname{tr}$ \\
\hline 14 & 1046 & $(2 E)$-octenal & 0.26 & 69 & 1526 & italicene ether & 0.40 \\
\hline 15 & 1059 & cis-sabinene hydrate & 0.02 & 70 & 1536 & $(E)$ - $\alpha$-bisabolene & 0.22 \\
\hline 16 & 1061 & octanol & 0.02 & 71 & 1546 & elemicin & 0.35 \\
\hline 17 & 1094 & linalool & $\operatorname{tr}$ & 72 & 1566 & 3-methyl pentadecane & 0.22 \\
\hline 18 & 1097 & (E)-6-methyl-3,5-heptadien-2-one & 0.47 & 73 & 1572 & caryophyllene oxide & 0.40 \\
\hline 19 & 1100 & nonanal & 2.28 & 74 & 1592 & longiborneol & 0.83 \\
\hline 20 & 1140 & 4-keto-isophorone & 0.49 & 75 & 1600 & hexadecane & 0.27 \\
\hline 21 & 1157 & $(2 E)$-nonenal & 0.77 & 76 & 1626 & $\alpha$-acorenol & 9.36 \\
\hline 22 & 1171 & nonanol & $\operatorname{tr}$ & 78 & 1645 & himachalol & 0.75 \\
\hline 23 & 1177 & terpinen-4-ol & 3.41 & 79 & 1647 & $\alpha$-bisabolol oxide B & 0.33 \\
\hline 24 & 1182 & dill ether & 0.57 & 81 & 1661 & neo-intermedeol & 1.25 \\
\hline 25 & 1200 & dodecane & $\operatorname{tr}$ & 82 & 1667 & intermedeol & 0.91 \\
\hline 26 & 1206 & decanal & 1.06 & 83 & 1671 & 2-methyl hexadecane & 0.64 \\
\hline 27 & 1214 & $(2 E, 4 E)$-nonadienal & 0.46 & 84 & 1677 & 3-methyl hexadecane & 0.31 \\
\hline 30 & 1238 & carvenone & $\operatorname{tr}$ & 85 & 1692 & 1-heptadecene & 0.47 \\
\hline 31 & 1248 & $p$-anisaldehyde & 0.98 & 86 & 1700 & heptadecane & 1.84 \\
\hline 32 & 1257 & decanol & 0.69 & 87 & 1714 & pentadecanal & 0.31 \\
\hline 33 & 1287 & $(2 E, 4 Z)$-decadienal & 0.74 & 89 & 1793 & 1-octadecene & 0.63 \\
\hline 34 & 1300 & tridecane & 0.33 & 90 & 1800 & octadecane & 0.69 \\
\hline 35 & 1311 & $(2 E, 4 E)$-decadienal & 1.46 & 91 & 1843 & 6,10,14-trimethyl-2-pentadecanone & 15.83 \\
\hline 37 & 1338 & $\alpha$-longipinene & $\operatorname{tr}$ & 92 & 1893 & 1-nonadecene & 0.84 \\
\hline 38 & 1344 & eugenol & 0.22 & 93 & 1900 & nonadecane & 1.36 \\
\hline 39 & 1348 & $(2 E)$-undecenal & 0.37 & 94 & 1947 & isophytol & 0.19 \\
\hline 40 & 1360 & undecanol & 0.51 & 95 & 1968 & palmitic acid (C16) & $\operatorname{tr}$ \\
\hline 41 & 1367 & protoillud-6-ene & $\operatorname{tr}$ & 96 & 1994 & 1-eicosene & 0.85 \\
\hline 42 & 1373 & 3-methyl tridecane & $\operatorname{tr}$ & 97 & 2000 & eicosane & 0.81 \\
\hline 43 & 1400 & tetradecane $+\beta$-longipinene & 0.74 & 98 & 2094 & 1-heneicosene & 0.61 \\
\hline 44 & 1401 & 2-dodecanone & 0.85 & 99 & 2100 & heneicosane & 1.12 \\
\hline 45 & 1408 & $\alpha$-cis-belgamotene & 2.91 & 100 & 2156 & ethyl linoleate & 0.56 \\
\hline 46 & 1415 & $\beta$-cedrene & $\operatorname{tr}$ & 101 & 2194 & 1-docosene & 0.27 \\
\hline 47 & 1424 & $\beta$-gurjunene & 0.85 & 102 & 2200 & docosane & 0.34 \\
\hline 48 & 1428 & $\alpha$-trans-bergamotene & 1.11 & 103 & 2294 & 1-tricosene & 0.23 \\
\hline 51 & 1446 & geranyl acetone & 0.82 & 104 & 2300 & tricosane & 0.46 \\
\hline 52 & 1454 & 2-methyl tetradecane & 0.59 & 106 & 2395 & 1-tetracoseene & $\operatorname{tr}$ \\
\hline 53 & 1456 & $\alpha$-acoradiene & 0.92 & 107 & 2400 & tetracosane & 0.18 \\
\hline 54 & 1461 & 3-methyl tetradecane & 0.59 & 108 & 2500 & pentacosane & $\operatorname{tr}$ \\
\hline 55 & 1466 & $\beta$-chamigrene & 0.71 & & & & \\
\hline 57 & 1473 & $(E)$ - $\beta$-ionone & 0.54 & & & & \\
\hline 58 & 1477 & ar-curcumene $+\beta$-chamigrene & 1.70 & & & total & 92.41 \\
\hline
\end{tabular}


Table 2 Classification of the Components of Woods Oil from Prunus mume.

\begin{tabular}{lc}
\hline \multicolumn{1}{c}{ Component } & Peak area (\%) \\
\hline Aliphatic & \\
Monoterpenoid & 3.41 \\
alcohols & $\operatorname{tr}$ \\
ketones & 0.57 \\
miscellaneous & \\
Sesquiterpenoid & 20.25 \\
hydrocarbons & 13.42 \\
alcohols & 0.54 \\
ketones & 1.09 \\
miscellaneous & \\
Diterpenoids & 0.19 \\
alcohols & \\
Miscellaneous & 13.97 \\
hydrocarbons & 1.22 \\
alcohols & 7.88 \\
aldehydes & 18.88 \\
ketones & $\operatorname{tr}$ \\
acids & 0.56 \\
esters & 3.84 \\
miscellaneous & \\
Aromatic & 0.22 \\
alcohol & 4.85 \\
aldehyde & $\operatorname{tr}$ \\
hydrocarbon & 1.48 \\
miscellaneous & 7.59 \\
unknown & \\
\hline tr: trace $<0.03 \%$. & \\
\hline
\end{tabular}

\section{Acknowledgment}

This work was supported by "High-Tech Research Center" project for Private Universities: matching fund subsidy from MEXT(Ministry of Education, Culture, Sports, Science and Technology), 2004-2008.

\section{References}

1. Y. CHUDA, H. ONO, M. OHNISHI-KAMEYAMA, K. MATSUMOTO, T. NAGATA and Y. KIKUCHI, Mumefural, Citric Acid Derivative Improving Blood Fluidity from Fruit-Juice Concentrate of Japanese Apricot (Prunus mume Sieb. et Zucc), J. Agric. Food Chem., Vol. 47, 828-831 (1999).

2. W. TAKATSUJi, S. IKEMOTO, H. SAKAGUCHI and H. MINAMI, Development of a New Type of Umeshu Using Immobilized Growing Yeast Cells, Nippon Jozo Kyokaishi, Vol. 87, 533-537 (1992).

3. M. MIYAZAWA, T. YAMADA and H. UTSUNOMIYA, Suppressive Effect of the SOS-inducing Activity of Chemical Mutagen by Citric Acid Esters from Prunus mume Sieb. Et Zucc. Using the Salmonella typhimurium TA1535/PSK1002 UMU Test, Nat. Prod. Res., Vol. 17, 319-323 (2003).

4. H. KAMEOKA and C. KITAGAWA, The Consituents of the Fruits of Prunus Mume Sieb. et Zucc., Nippon Nogei Kagaku Kaishi, Vol. 50, 389-393 (1976).

5. C. YUKAWA, Y. IMAYOSHI and H. IWABUCHI, Volatile Components of Japanese Apricot (ume, Prunus mume Sieb. et Zucc.) (I), Foods Food Ingredients J., Vol. 166, $76-79$ (1995).

6. C.C. CHEN, M.C. KUO, S.E. LIU and C.M. WU, Volatile Components of Salted and Pickled Prunes (Prunus mume Sieb. et Zucc.), J. Agric. Food Chem., Vol. 34, 140-144 (1986).

7. T. ARIKAWA, S. OHSHIMA and H. TAKAGAKI, Aroma Substances in Umeshu (Japanese apricot liqueur) and Their Changes during Storage, Nippon Kaseigaku Kaishi, Vol. 48, 295-301 (1997).

8. M. ISHIDA, Volatile Components of Umeboshi, Koryo, Vol. 211, 139-156 (2001).

9. M. HASEGAWA, Flavonoids of Various Prunus Species. X. Wood Constituents of Prunus tomentosa, Shokubutsugaku Zasshi, Vol. 82, 458-461 (1969).

10. M. HASEGAWA, Flavonoids of Various Prunus Species. IX. Two New Flavonoid Glycosides from the Wood of Prunus mume, Shokubutsugaku Zasshi, Vol. 82, 148-154 (1969).

11. M. HASEGAWA, Flavonoids of Various Prunus Species. VIII. Flavonoids in the Wood of P. mume, J. Org. Chem., Vol. 24, 408-409 (1959).

12. R.P. ADAMS, Identification of Essential Oil Components by Gas Chromatography/ Quadrupole Mass Spectroscopy., Allured Publ. Corp., Carol stream, IL, USA (2001). 